\title{
CYPRODINIL, PYRIMETHANIL, PROPINEB E TRIFLOXYSTROBIN NO CONTROLE DA SARNA DA MACIEIRA
}

\section{CYPRODINIL, PYRIMETHANIL, PROPINEB AND TRIFLOXYSTROBINON APPLE SCAB CONTROL}

\author{
Onofre BERTON ${ }^{1}$ \\ Louise Larissa MAY-DE MIO² \\ Hellen Aparecida Arantes dos SANTOS ${ }^{3}$
}

\begin{abstract}
RESUMO
Estudou-se o efeito dos fungicidas cyprodinil, pyrimethanil, propineb e trifloxystrobin no controle da sarna da macieira na cultivar Gala. A eficiência dos fungicidas ficou maior com os sistêmicos, mas determinada pela dosagem. Nos frutos, o efeito sobre a doença variou entre $78 \%$ e $100 \%$ de controle em relação à testemunha. Nas folhas, a sarna foi controlada pelos fungicidas com eficiência igual ou superior a $70 \%$ em relação à testemunha, entretanto nenhum fungicida proporcionou controle de $100 \%$. De dezembro a abril a incidência da sarna nas folhas da testemunha foi de 0 a $99 \%$ atingindo uma severidade média de 14 lesões/folha. O número médio de lesões de sarna/folha dos tratamentos com fungicidas diferiu significativamente da testemunha e a média de controle foi superior a $80 \%$. Não foi observado efeito fitotóxico sobre ramos e folhas da cultivar Gala, também não foi observado "russeting" sobre os frutos pela ação dos fungicidas.
\end{abstract}

Palavras-chave: Controle químico, fungicidas, Malus domestica, Venturia inaequalis.

\begin{abstract}
Effects of the fungicides cyprodinil, pyrimethanil, propineb and trifloxystrobin were studied for apple scab control on cv "Gala". Effect of the fungicides was evidenced for systemic compounds and determined by the dose used. On fruits the effect upon apple scab varied between $78 \%$ and $100 \%$. On leaves, fungicides controlled apple scab with at least $70 \%$ efficiency and none of the fungicides controlled $100 \%$. From December to April the incidence of apple scab disease in the control treatment ranged from $0,0 \%$ to $99 \%$ reaching severity of 14 lesions/leave. The average number of lesions per leaf in all treatments with fungicides was significantly different from control and the mean control for apple scab was superior to $80 \%$. Neither phytotoxicity over spurs and leaves nor russeting on fruits were observed after ten fungicide sprays.
\end{abstract}

Key-words: chemical control, fungicides, Malus domestica, Venturia inaequalis.

${ }^{1}$ Engenheiro Agrônomo, Ph.D. em Fitopatologia, Pesquisador da Epagri - Estação Experimental de Caçador.

${ }^{2}$ Engenheira Agrônoma, Doutora em Fitopatologia, Professora do Departamento de Fitotecnia e Fitossanitarismo, da UFPR. Rua dos Funcionários 1540, 80035-050, Curitiba-PR, maydemio@ufpr.br. Autora para correspondência.

${ }^{3}$ Engenheira Agrônoma. 
BERTON, O. et al. Cyprodinil, Pyrimethanil...

\section{INTRODUÇÃO}

A cultura da macieira (Malus domestica Bork.), no sul do Brasil atinge uma grande área cultivada, aproximadamente 35,5 mil hectares para a safra 04/ 05. Sua produção vem se ampliando a cada ano, alcançando na safra 04/05 846,4 mil toneladas (VIEIRA, 2006). A sarna (Venturia inaequalis Cke. Wint.) é considerada a doença de maior ocorrência na macieira, no mundo (WESTWOOD, 1982). No Brasil, ela ocorre em todos os Estados produtores, mas nas regiões mais altas e frias suas conseqüências são mais prejudiciais (BLEICHER, 1986), podendo causar danos superiores a $80 \%$ na produção (BONETI et al., 2002). Os sintomas da sarna são bastante típicos nos frutos e nas folhas. Em ambas as superfícies das folhas, surgem, inicialmente, pequenas manchas de cor verde-oliva que vão tornando-se acinzentadas. As lesões são de formato circular, podendo coalescer ou permanecer isoladas, espalhando-se por toda a superfície foliar. No outono, após a queda natural das folhas e a morte das suas células, o micélio penetra profundamente no tecido do hospedeiro e inicia-se a formação do pseudotécio. Para que isso ocorra, o anterídio e o ascogônio precisam ser oriundos de indivíduos distintos, uma vez que o fungo é tipicamente heterotálico. Conforme KATSURAYAMA (2005), o pseudotécio forma-se durante o outono e inverno, sendo a temperatura ideal de $4{ }^{\circ} \mathrm{C}$, não havendo formação em temperaturas igual ou superior a 15 ${ }^{\circ} \mathrm{C}$. A umidade também é um fator limitante na formação dos pseudotécios, que não ocorre em folhas muito secas.

A principal medida de controle da sarna é o uso de fungicidas, pois as principais cultivares (Gala, Fuji e Golden Delicious), são muito suscetíveis. No Paraná a região de Palmas é a mais fria e também onde ocorre, com mais freqüência, epidemias da doença tanto na fase de florada, evidenciando sintomas nos frutos em formação, como também provocando a sarna tardia nas folhas que ocorre após a colheita (BERTON, 2002). Este tipo de sarna tem aumentado nas últimas safras quando a temperatura diminue no verão. O problema é que a doença se não for bem controlada deixa a folha com aspecto enegrecido, impedindo a fotossíntese, e mantém o inóculo para o próximo ciclo. A sarna é uma doença muito estudada no mundo todo, porém ainda são poucas as pesquisas de opções de fungicidas no Brasil, estas pesquisas são de extrema importância para redução de perdas da cultura. Os fungicidas sistêmicos são interessantes para o controle desta doença, pois inibem, seletivamente, processos metabólicos específicos, em grupos restritos de fungos, como a síntese dos ácidos nucléicos, de proteínas, de lipídios, de quitina, de ergosterol e na respiração celular (COSTA, 1993), e podem conter o avanço da doença no campo, entretanto o uso de fungicidas protetores é importante dentro de um sistema de manejo da resistência do patógeno aos fungicidas no campo. Neste ponto é importante salientar o benefício para o produtor e para o sistema de produção ter opções de fungicidas de diferentes grupos para o correto manejo da doença.

O presente trabalho teve como objetivo avaliar o efeito dos fungicidas cyprodinil, pyrimethanil (em três dosagens), propineb e trifloxystrobin (em duas dosagens) comparados com testemunha sem aplicação de fungicidas para controle da sarna nos frutos e nas folhas de macieira.

\section{MATERIAL E MÉTODOS}

O experimento foi desenvolvido no Município de Palmas - PR, região que se situa a uma latitude 2629'03“ sul e a uma longitude 51ำ59'26" oeste, estando a uma altitude de 1035 metros, com clima predominante $\mathrm{Cfb}$, segundo a Classificação de Köppen, em um Latossolo Distrófico, durante a safra $2004 / 05$. O delineamento experimental foi blocos ao acaso com oito tratamentos (Tabela 1) e quatro repetições sendo que cada parcela era composta de cinco plantas da cultivar Gala, porta enxerto MM106, em espaçamento de $4 \times 1 \mathrm{~m}$, em um pomar de 6 anos. A partir de $27 / 12$ os produtos foram aplicados com pulverizador costal manual marca Jacto, com ponteira de três bicos, operando a uma pressão em torno de $515 \mathrm{kPa}$, até o ponto de início de escorrimento com intervalos de sete a dez dias conforme o regime de precipitação. Foram aplicados dez litros da calda fungicida para as quatro repetições, equivalendo a um volume de $1200 \mathrm{~L} \mathrm{ha}^{-1}$. No período de dezembro a março foram realizadas dez pulverizações no experimento.

TABELA 1 - Fungicidas com formulação e concentração utilizados no experimento para controle de sarna da macieira em Palmas-PR, 2004/05.

\begin{tabular}{|c|c|c|c|c|}
\hline Tratamento & Ingrediente ativo & Formulação & $\begin{array}{l}\text { Concentração } \\
\text { produto comercial } \\
\left(\mathrm{ml} 100 \mathrm{~L}^{-1}\right)\end{array}$ & $\begin{array}{c}\text { Concentração } \\
\text { ingrediente ativo } \\
\left(\mathrm{g} 100 \mathrm{~L}^{-1}\right)\end{array}$ \\
\hline $\begin{array}{l}\text { Unix } \\
\text { Mythos 1 } \\
\text { Mythos } 2 \\
\text { Mythos } 3 \\
\text { Antracol } \\
\text { Flin 1 } \\
\text { Flin 2 }\end{array}$ & $\begin{array}{l}\text { Cyprodinil } \\
\text { Pyrimethanil } \\
\text { Pyrimethanil } \\
\text { Pyrimethanil } \\
\text { Propineb } \\
\text { Trifloxystrobin } \\
\text { Trifloxystrobin }\end{array}$ & $\begin{array}{l}75 \mathrm{WG} \\
300 \mathrm{SC} \\
300 \mathrm{SC} \\
300 \mathrm{SC} \\
70 \mathrm{PM} \\
500 \mathrm{WG} \\
500 \mathrm{WG}\end{array}$ & $\begin{array}{l}20 \\
100 \\
125 \\
150 \\
200 \\
7,5 \\
10\end{array}$ & $\begin{array}{c}15 \\
30 \\
37,5 \\
45 \\
140 \\
3,75 \\
5\end{array}$ \\
\hline
\end{tabular}


Até dezembro, a área escolhida não tinha recebido tratamento químico e estava com uma alta incidência de sarna nos frutos, entretanto a sarna ainda não estava presente nas folhas. Neste contexto, foi avaliada a ação dos produtos nos frutos já infectados de dezembro até a colheita (fevereiro) e ação preventiva nas folhas (que estavam inicialmente sem sintoma da doença) de dezembro a abril.

Para avaliação da doença no fruto foram marcados dois ramos por planta central em cada parcela de cinco plantas e anotados o número de frutos por ramo e a incidência da doença na instalação do experimento, antes da primeira aplicação dos fungicidas. Na colheita, os frutos destes ramos foram colhidos separadamente e avaliados. Com a relação entre a doença no início e após as pulverizações calculou-se a porcentagem de controle da sarna.

As avaliações de incidência e severidade da sarna nas folhas foram realizadas em três datas: no início do experimento (em dezembro), em fevereiro (após cinco aplicações) e no final do experimento, em abril (após dez aplicações). Em cada data foram coletadas cinqüenta folhas por parcela, sendo vinte e cinco de cada lado daplanta central e determinada a incidência da doença (número de folhas com sarna). A severidade da sarna foi avaliada contando-se o número de lesões por folha devido às lesões serem homogenias em tamanho e para ter uma maior precisão na avaliação.

O progresso da doença no fruto foi determinado por meio de três avaliações sendo a primeira por ocasião da instalação, em 22/12/04, a segunda em 14/01/05 e a terceira em 02/02/05, na colheita. $\mathrm{Na}$ colheita foi determinado o peso de cinqüenta frutos por parcela para cada tratamento.

As análises dos resultados dos ensaios foram realizadas com o programa SASM-Agri (CANTERI et al., 2001) utilizando o teste estatístico Scott-Knott, que é um método de agrupamento usado como alternativa em que procedimentos de comparações múltiplas são recomendados, com a característica de não apresentar ambigüidade nos resultados (SILVA et al., 1999).

\section{RESULTADOS E DISCUSSÃO}

\section{Efeito de fungicidas no controle da sarna em frutos}

O tratamento testemunha iniciou com $50 \%$ de doença e houve um aumento de aproximadamente $67 \%$ de dezembro a fevereiro (Figura 1) enquanto a maioria dos fungicidas demonstrou efeito positivo impedindo que a doença aumentasse com base na primeira avaliação. Os fungicidas, cyprodinil e trifloxystrobin na menor dosagem não tiveram efeito a partir da segunda avaliação. Considerando o aumento máximo da doença como sendo o da testemunha, a Figura 2 mostra o porcentual de controle relativo dos tratamentos.

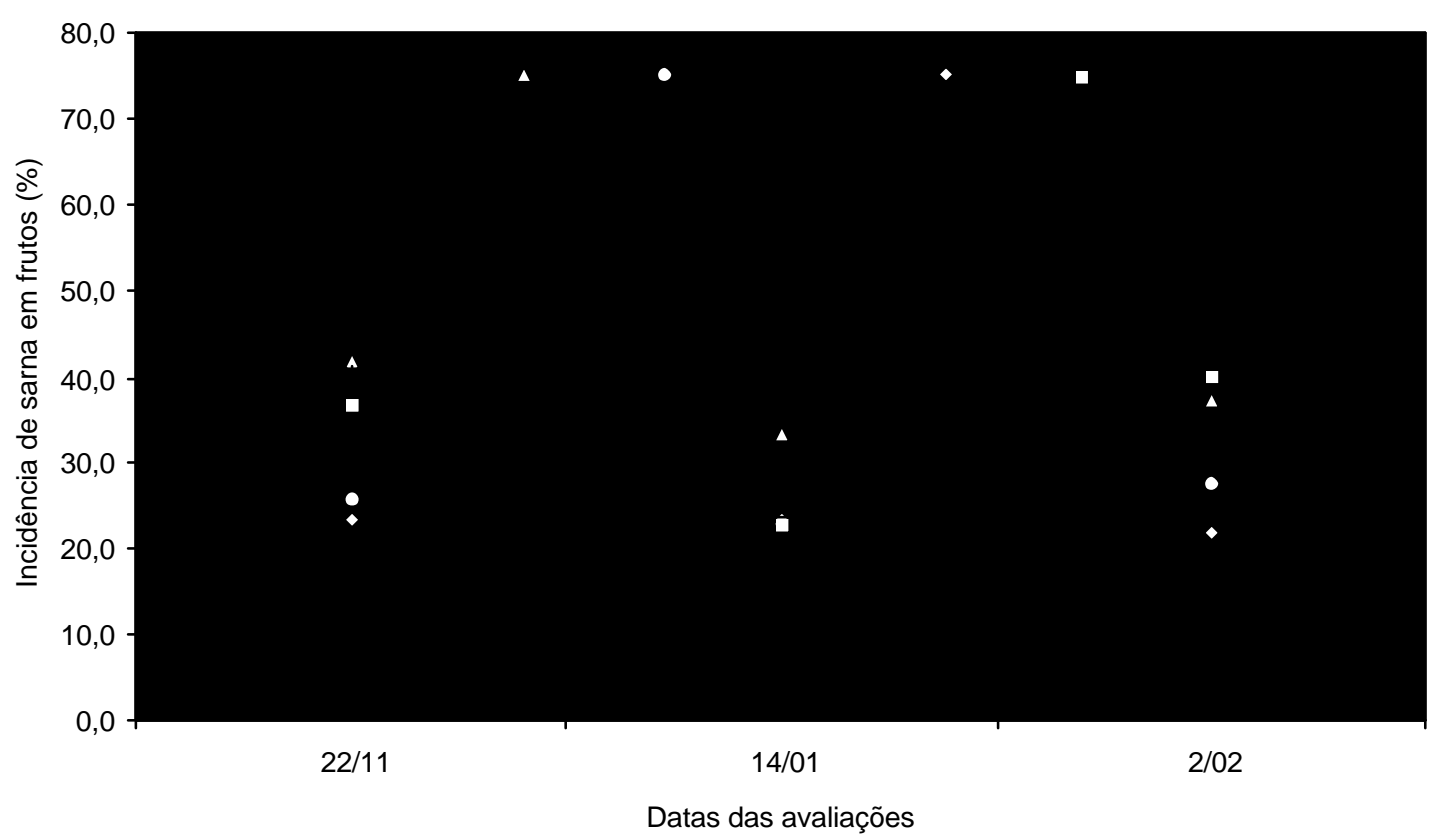

FIGURA 1 - Progresso da sarna da macieira em frutos da cultivar Gala, em três datas de avaliação, em diferen-

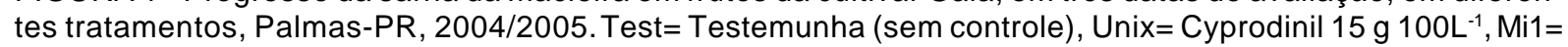

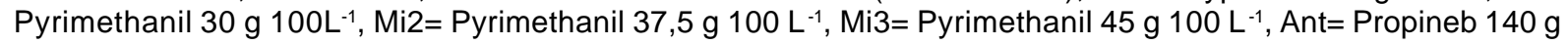
$100 \mathrm{~L}^{-1}$, Flin1 = Trifloxystrobin 3,75 g $100 \mathrm{~L}^{-1}$, Flin2=Trifloxystrobin $5 \mathrm{~g} 100 \mathrm{~L}^{-1}$. 
BERTON, O. et al. Cyprodinil, Pyrimethanil...

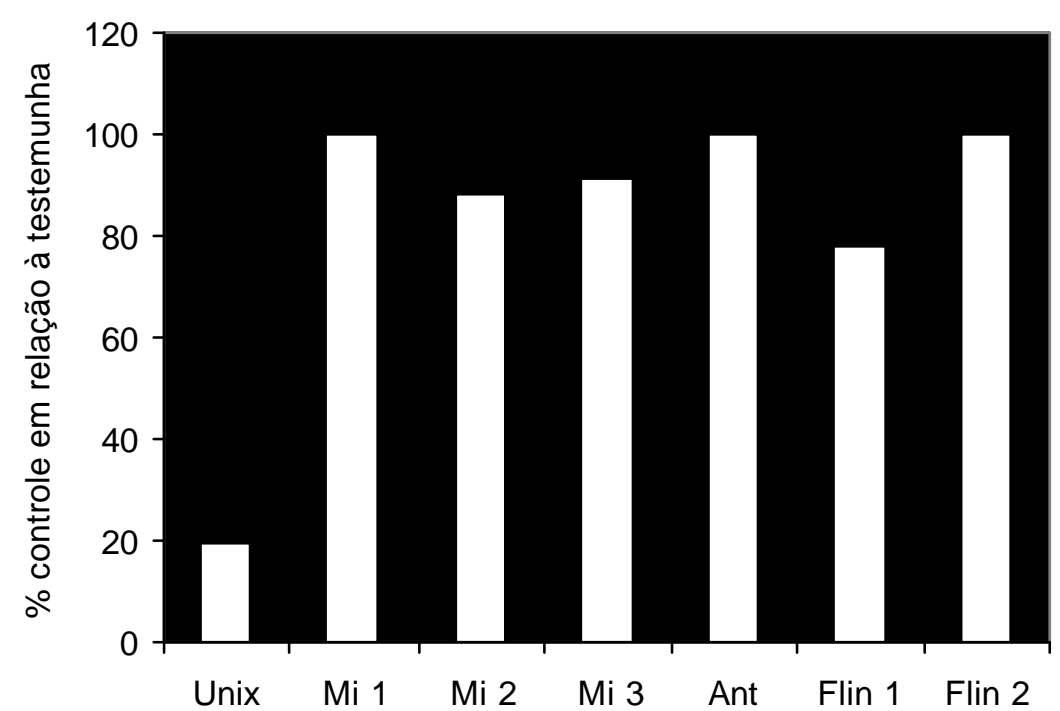

FIGURA 2 - Porcentagem de controle da sarna em frutos de Gala após cinco aplicações com os diferentes fungicidas, Palmas-PR, 2004/2005. Test= Testemunha (sem controle), Unix= Cyprodinil $15 \mathrm{~g}$

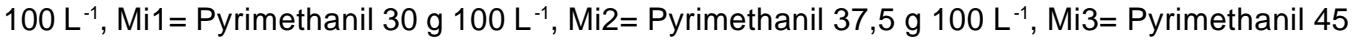

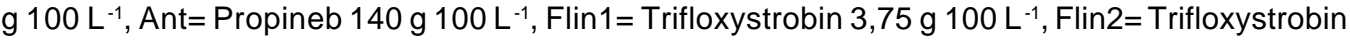
$5 \mathrm{~g} 100 \mathrm{~L}^{-1}$.

\section{Avaliação dos frutos na colheita}

Os cinqüenta frutos colhidos por parcela foram avaliados quanto à incidência de sarna e peso dos frutos. Todos os tratamentos com fungicidas diferiram significativamente da testemunha com relação à incidência e severidade de sarna, de acordo com a análise estatística ao nível de $5 \%$ de significância. A menor percentagem de controle da sarna nos frutos, em relação à testemunha foi conseguida com a dosagem única de cyprodinil, com valor aproximado de $20 \%$. Os demais tratamentos mostraram percentagens de controle entre 78 e $100 \%$. Nesta fase não foram observados sintomas de fitotoxidez "russeting" nos frutos em qualquer dos tratamentos.

\section{Efeito preventivo de fungicidas no controle da sarna nas folhas.}

A doença nas folhas progrediu de dezembro a abril (Tabela 2), sendo que a curva da doença na testemunha saiu de $0 \%$ de incidência e chegou a $99 \%$ em abril enquanto nas folhas se formaram em média 14 lesões de dezembro a fevereiro (40 dias). A severidade em abril não pôde ser estimada, pois na testemunha as folhas estavam escurecidas e impossível de diferenciar as lesões. Para efeito de análise o valor de fevereiro da testemunha foi repetido em abril. 
BERTON, O. et al. Cyprodinil, Pyrimethanil...

TABELA 2 - Ação dos fungicidas cyprodinil, pyrimethanil e trifloxystrobin para controle da sarna nas folhas após cinco e dez aplicações em relação aos parâmetros incidência e severidade, de dezembro/2004 a abril/2005.

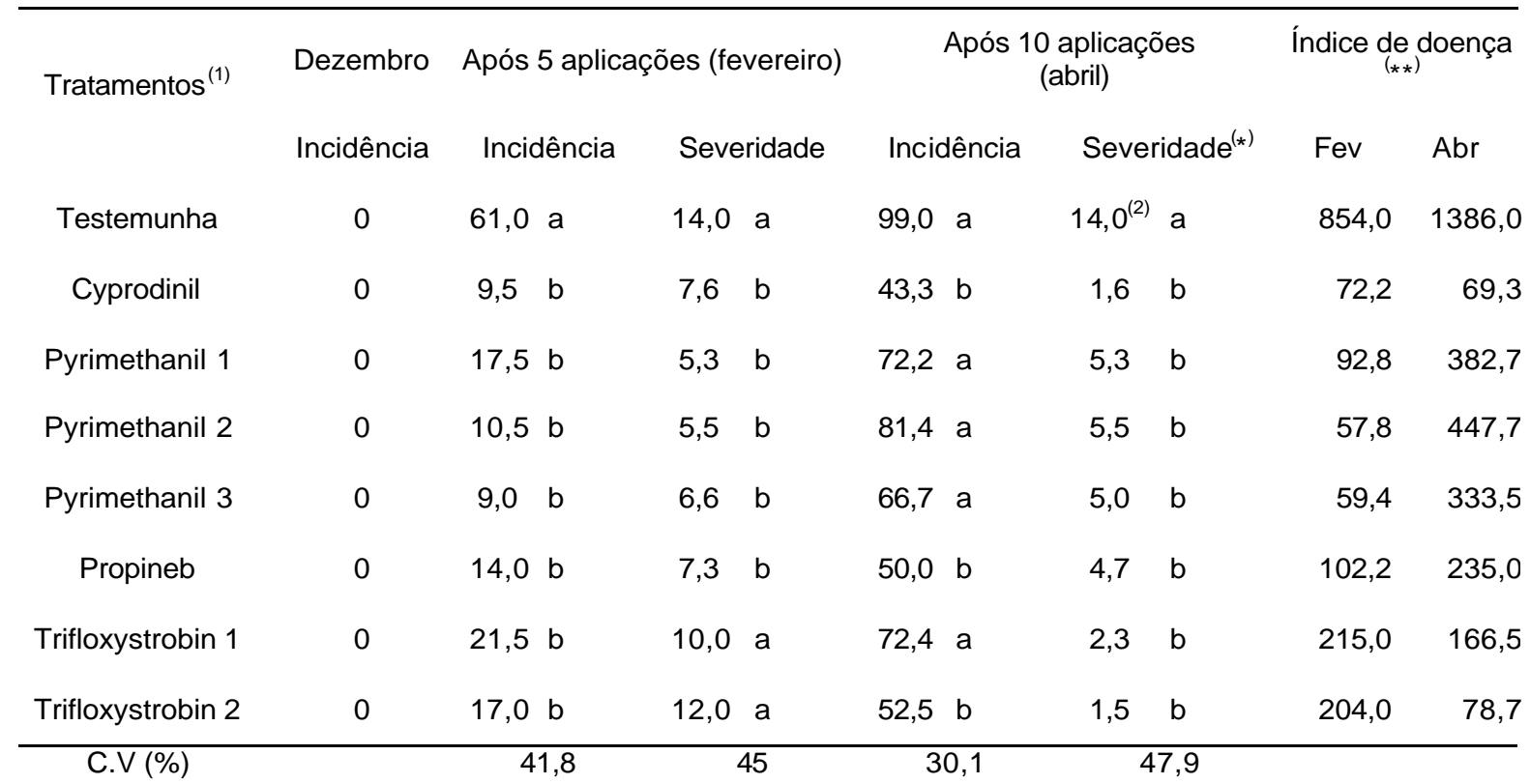

(1) Médias seguidas pela mesma letra, nas colunas, não diferem entre si pelo teste de Scott-Knott a $5 \%$ de probabilidade.(2) Valor repetido da primeira avaliação, pois as folhas estavam totalmente pretas, sem condições para diferenciar as lesões devido a alta severidade de sarna.$^{(*)}$ com arc sen raiz $x / 100 .{ }^{\left({ }^{* *}\right)}$ considerado a inc*sev. (Inc=Incidência média em 50 folhas por parcela, Sev= Severidade em número de lesões/folha, média de dez folhas por parcela).

A incidência da sarna nas folhas tratadas estava baixa e diferente estatisticamente da testemunha na avaliação de fevereiro, independentemente dos tratamentos, entretanto em abril os fungicidas que se destacaram foram o cyprodinil, propineb e trifloxystrobin na maior dosagem. Quanto à severidade, todos os tratamentos diferiram da testemunha e o percentual de controle relativo foi alto com valores em torno de $80 \%$ para todos os tratamentos em todas as avaliações (Figura 3).

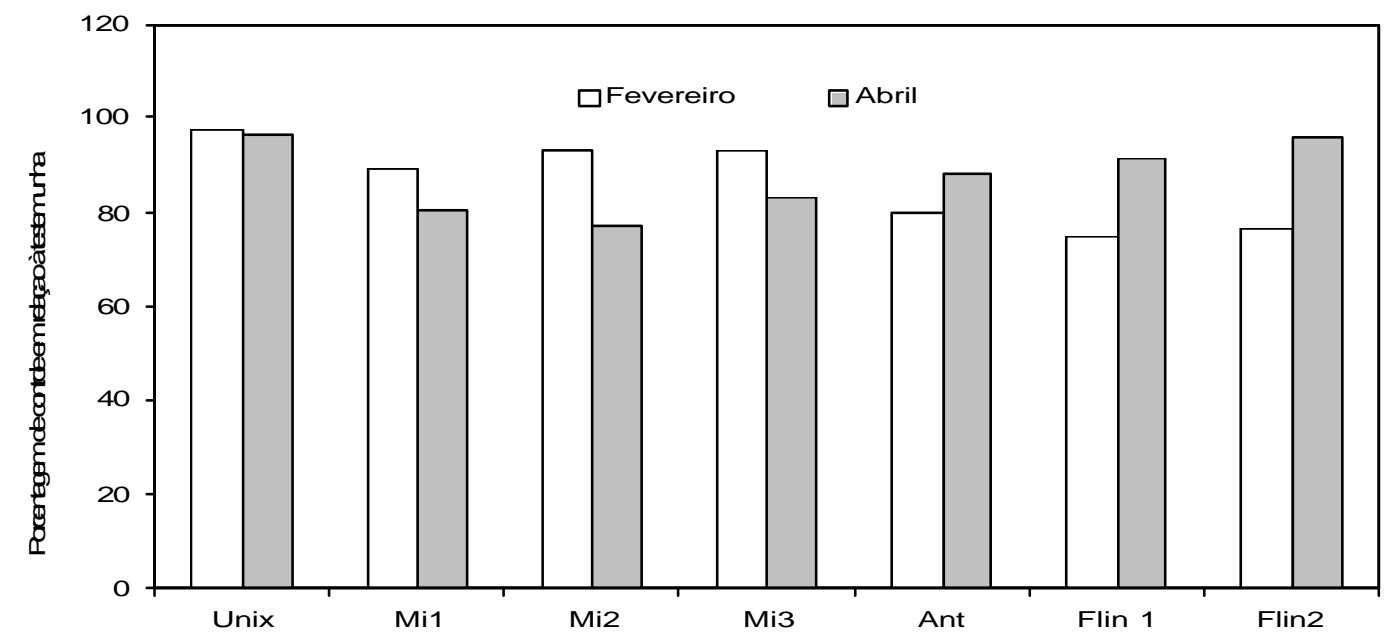

FIGURA 3 - Porcentagem de controle da sarna nas folhas da cultivar Gala em relação à testemunha sem controle com o uso preventivo de fungicidas. Palmas-PR, 2004/2005. Test= Testemunha (sem

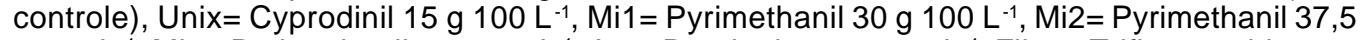

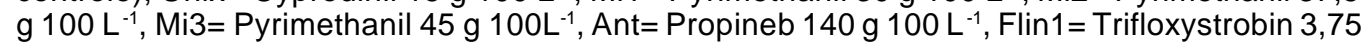
g $100 \mathrm{~L}^{-1}$, Flin2= Trifloxystrobin $5 \mathrm{~g} 100 \mathrm{~L}^{-1}$. 
No Sul do Brasil, o período crítico para a ocorrência da sarna inicia-se com a brotação da macieira, coincidindo com setembro/outubro, e prolonga-se até o final do mês de novembro a meados de dezembro, quando cessa a liberação de ascósporos (MELZER e BERTON, 1998). Nesse período, ocorrem rápida expansão foliar e o desenvolvimento dos ramos terminais. Após este período pode ainda ocorrer um aumento da doença nas folhas e o progresso da epidemia se estende até o período de queda das folhas, prejudicando a produção para o próximo ciclo. Como pode ser confirmado neste trabalho na região de Palmas é importante o controle da doença na folha, pois a epidemia aumenta até o período de início de queda das folhas.

A principal medida de controle da sarna é o uso de fungicidas, pois as principais cultivares são muito suscetíveis a esta doença. Os fungicidas sistêmicos inibem, seletivamente, processos metabólicos específicos, em grupos restritos de fungos, como a síntese dos ácidos nucléicos, de proteínas, de lipídios, de quitina, de ergosterol e na respiração celular (COSTA, 1993) e podem conter o avanço da doença no campo.

A grande capacidade seletiva e especificidade dos fungicidas sistêmicos, que lhes permite aumentar sua eficiência em relação aos fungicidas protetores e erradicantes, é, ao mesmo tempo, a causa de sua vulnerabilidade. No presente trabalho verificamos
BERTON, O. et al. Cyprodinil, Pyrimethanil... ação positiva dos fungicidas, entretanto, a recomendação para este fim deve ser cuidadosa para evitar uma pressão de seleção de raças resistentes. Os fungos, por apresentarem grande variabilidade genética, podem tornar-se resistentes a fungicidas de ação específica. O problema da resistência depende, em grande parte, da pressão de seleção que é exercida pelo aumento na freqüência de aplicação, isto é, pela inadequada aplicação de fungicidas. Essa pressão é uma função da extensão e duração da exposição, sendo tanto maior quanto maior a área tratada com apenas um ingrediente ativo específico e quanto maior a dosagem e o número de aplicações (MARSHA, 1977). Neste ponto é importante salientar o benefício para o produtor e para o sistema de produção ter opções de fungicidas, de diferentes grupos para o correto manejo da doença, o qual foi o objetivo inicial deste trabalho.

\section{CONCLUSÕES}

Os fungicidas cyprodinil, propineb e trifloxystrobin (maior dosagem) aplicados preventivamente reduzem a incidência da sarna da macieira em folhas.

Os fungicidas cyprodinil, pyrimethanil e propineb, aplicados preventivamente, reduzem a severidade da sarna da macieira em folhas em todas dosagenstestadas.

\section{REFERÊNCIAS}

1. BERTON, O. Ontogenic resistance, use of DMI fungicides and late season scab of apples. Ithaca, USA, 2002. $62 \mathrm{f}$. Dissertation (Doctorate) - Cornell University.

2. BLEICHER, J. Doenças da macieira. In: EMPASC. Manual da cultura da macieira. Florianópolis, 1986. p. 381-391.

3. BONETI, J.I. DA S.; KATSURAYAMA, Y.; BLEICHER, J. Doenças fúngicas foliares. In: A cultura da macieira. Florianópolis: Epagri, 2002. p. 527-537.

4. CANTERI, M.G.; ALTHAUS, R.A.; VIRGENS FILHO, J.S.; GIGLIOTI, E.A.; GODOY, C.V. SASM-Agri - Sistema para análise e separação de médias em experimentos agrícolas pelos métodos Scott-Knott, Tukey e Duncan. Revista Brasileira de Agrocomputação, Ponta Grossa, v. 1, n. 1, p. 18-24, 2001.

5. COSTA, I.F.D. Fitopatologia geral. Apostila de aulas teóricas. Cruz Alta: Universidade de Cruz Alta, 1993. 79 p.

6. KATSURAYAMA, Y. Doenças da macieira (Malus domesticaBork.). In: ENCONTRO NACIONAL SOBRE FRUTICULTURA DE CLIMA TEMPERADO, Fraiburgo, 2005. Anais . Caçador: EPAGRI, 2005. p. 241-257.

7. MARSHA, R.W. Systemic fungicides. 2. ed. London: Longeman, 1977. 401 p.

8. MELZER, R.; BERTON, O. Sistema de alerta para o controle da sarna da macieira. Florianópolis: Empasc, $1989.75 \mathrm{p}$.

9. SILVA, A.C.F.; ROSA, C.R.A.; MELO, I.S. Sensibilidade de isolados de Trichoderma spp. a benomil e iprodione. Revista Científica do Centro de Ciências Rurais, Santa Maria, v. 3, n. 29, p. 395-399, 1999.

10. VIEIRA, L.M. Síntese anual da agricultura de Santa Catarina. Florianópolis: CEPA-Epagri. Disponível em:< http:// cepa.epagri.sc.gov.br> Acesso em 20 DEZ2006.

11. WESTWOOD, M.N. Plagas y enfermedades. In: WESTWOOD, M.N. Fruticultura de zonas templadas. Madrid: Mundi Prensa, 1982. p.367-397.

Recebido em 06/11/2006 Aceito em 14/05/2007 